\title{
VARIA
}

\section{LA GÉNÉTIQUE AU MUSÉE : FIGURES ET FIGURANTS DU DÉBAT PUBLIC}

\author{
Igor Babou et Joëlle Le Marec ${ }^{1}$
}

Il existe un intense débat social autour des enjeux de la génétique et surtout des applications industrielles et médicales de la génomique. Les acteurs de ce débat s'expriment publiquement et sont bien identifiés, tant en France que dans le monde : associations et ONG, syndicats, partis politiques, chercheurs, juristes, groupes de l'industrie agroalimentaire, pharmaceutique ou bio-médicale, comités d'éthique, etc. Les expositions consacrées à la génétique sont remarquablement nombreuses depuis quelques années et cette multiplication témoigne à sa manière de l'intensité du débat. Elles réfèrent d'ailleurs assez souvent à l'existence de ce débat public et aux types d'arguments supposés s'y échanger. En analysant comment les expositions représentent ce débat public, nous nous attacherons à comprendre certains des processus socio-discursifs qui y opèrent.

Parmi l'ensemble des acteurs individuels ou collectifs pouvant inscrire leur identité et porter leurs arguments dans le discours de l'exposition, nous avons choisi d'étudier les formes de mobilisation du public à travers sa mise en scène dans le discours de l'exposition. L'analyse de l'énonciation permettra de repérer la manière dont le discours témoigne de rapports de légitimité existant soit entre acteurs

1 ENS Lettres et Sciences humaines.

Recherches en communication, $\mathrm{n}^{\circ} 20$ (2003). 
au sein de l'espace médiatique, soit entre le média et son extériorité. Mais une analyse énonciative centrée sur le texte de l'exposition ne nous parait pas suffisante pour rendre compte du fonctionnement socio-discursif de l'exposition en tant que média. En effet, il n'existe pas de "public" si on entend par-là un acteur collectif incarné dans un groupe doté de représentants légitimes, qui pourrait revendiquer la possibilité d'intervenir sur le contenu d'une exposition. La critique des expositions est quasiment inexistante et ne dispose pas d'un champ éditorial structuré comme celui de la télévision par exemple. En revanche le statut de membre du public est parfaitement assumé individuellement, par un grand nombre de personnes qui se sentent liées aux institutions productrices d'expositions. Par exemple, au cours des enquêtes sociologiques sur le public, ce statut s'actualise dans l'interaction avec le chercheur en sciences sociales qui est reconnu comme "représentant" des institutions savantes ${ }^{1}$. Ceci rend possible la production d'un discours scientifique sur le public et la mise à jour d'un contrat de communication entre l'institution et le public, mais dans un tout autre espace que celui de l'exposition. La sémiotique du "texte" de l'exposition ne peut pas rendre compte de ce type de phénomène, qui ne s'inscrit nulle part ailleurs que dans l'enregistrement des situations d'interactions, en particulier avec le sociologue.

Trois instances pourraient donc avoir vocation à représenter le public dans l'espace médiatique que constitue l'exposition : des groupes constitués (associations d'amis des musées, d'abonnés, mouvements d'opinions, etc.), le champ éditorial de la critique (dont on vient de dire qu'il est presque inexistant), et les sciences sociales productrices d'un savoir en principe légitime sur le public (à la fois au plan empirique, et sur la notion théorique de "public").

Nous allons poser trois types de questions :

- Y a t-il une critique des expositions de science qui pourrait modifier l'équilibre des rapports de légitimité et influencer les formes et le contenu des discours?

- Existe-t-il des formes d'organisation collective de membres du public qui revendiqueraient la possibilité de porter leurs arguments dans le discours de l'exposition?

1 J. LE MAREC, "Le musée à l'épreuve des thèmes sciences et société", Quaderni $n^{\circ} 46,2002$. 
- La représentation du débat public dans le discours de l'exposition tient-elle compte des connaissances produites par les sciences sociales au sujet du public ?

\section{Critiques d'expositions : une étonnante absence}

Si les expositions d'art bénéficient d'un champ éditorial qui leur consacre des rubriques régulières (magazines spécialisés, presse quotidienne, suppléments culturels, etc.) on serait bien en peine de trouver un support ou une rubrique qui constituerait l'espace d'une critique des expositions scientifiques et techniques, et plus largement, des expositions thématiques. Même dans le cas des expositions artistiques, c'est moins la muséographie qui est commentée, que les œuvres et leur sélection, très rarement, leur accrochage. Dans la tradition de l'esthétique et de l'histoire de l'art, il s'agit d'une critique de la production plastique et non du dispositif. Dans le cas d'expositions de points de vue, destinées à produire un discours plus qu'à exposer des œuvres ou des objets, et qui mettent en œuvre une pluralité d'expôts dans un environnement scénographié, si l'on écarte les échanges au sein même de la communauté professionnelle, le commentaire du dispositif est plutôt pris en charge dans le registre académique des sciences sociales, par des sémioticiens, linguistes, etc. C'est ainsi qu'une des seules chroniques véritablement consacrées à une analyse muséographique a été faite par André Desvallées ${ }^{1}$ dans une revue scientifique, Publics et Musées. C'est un support académique spécialisé, interne au champ muséologique, qui a assumé à cette époque le commentaire critique, et non un média externe à l'institution. Pourtant, les expositions à caractère scientifique et technique ont fait l'objet d'une critique régulière au début du siècle, notamment dans les pages de L'Illustration ${ }^{2}$. L'absence de champ critique ne constitue donc pas une caractéristique en soi des expositions, mais relève d'un état historiquement situé des rapports entre différents médias.

1 Conservateur, André Desvallées a été membre fondateur du mouvement de la Nouvelle Muséologie. Il a coordonné l'édition de Vagues, anthologie de la nouvelle muséologie, Mâcon, Éditions W. MNES, 1994.

2 Nous avons dépouillé L'Illustration des années 1922 à 1925 : on y trouve un grand nombre d'articles consacrés à des expositions à caractère technique : expositions coloniales, expositions d'arts appliqués, etc. 


\section{L'organisation collective des membres du public : "pas de lobbying d'Attac, par pitié ?""}

Le public est jugé toujours trop volatile et imprévisible quand il est pensé par les acteurs institutionnels en terme d'audience, de phénomène additif et statistique, de foule. Mais dans le même temps, dès que des individus s'organisent en tant que membres du public pour se légitimer comme interlocuteurs impliqués dans le dialogue avec l'institution, ils ne sont pas reconnus comme tels, et parfois même redoutés. Ainsi dans une réunion à laquelle nous participions avec des responsables politiques soucieux de promouvoir la culture scientifique, la proposition d'organiser un débat délibératif au sein d'un musée, pour poser celui-ci comme un espace public et d'argumentation, a provoqué ce cri du cœur qui résume bien la situation: "Pas de lobbying d'Attac, par pitié !". Quand une opinion organisée émerge au sein d'un public intéressé par les sciences, et qu'elle ne s'exprime pas dans les termes du rapport au savoir posé du point de vue de l'institution, elle semble disqualifiée et rabattue dans le registre de la manipulation politique par des groupes de pression : le "public" doit constituer une masse la plus importante possible, mais toujours identifiable à un pôle de la "réception". Même lorsque c'est l'institution elle-même qui suscite la constitution de groupes de publics organisés (associations d'abonnés, associations d'amis de musées), c'est souvent avec la volonté de distinguer un groupe de clients privilégiés, intéressés à l'usage d'une offre culturelle de ressources et de services. Dans le cas des musées d'art et d'histoire, les sociétés d'amis de musées sont des instances bénévoles qui se mettent au service des intérêts du musée, par exemple pour contribuer à l'acquisition des oeuvres'. Cependant les institutions ne souhaitent généralement pas que s'expriment des valeurs éventuellement contradictoires à la proposition muséale.

I Dans ce cas, les sociétés d'amis inscrivent leur appartenance à l'institution muséale par exemple à travers la citation de leur nom comme donateurs sur les cartels. Voir le Code d'éthique des amis et bénévoles de musées, Fédération mondiale des amis de musées : http://www.museumsfriends.org/frances/f_codigo.html 


\section{Les représentations du débat public dans l'exposition}

Pour décrire les deux précédentes instances de représentation du public, il faut tenir compte de phénomènes externes au discours de l'exposition. La troisième instance, celle des représentations du débat public dans l'exposition, amène quant à elle à se focaliser sur le discours. Pour autant il ne s'agit pas de revenir à des analyses de corpus clos sur eux-mêmes, mais de porter attention au dispositif ${ }^{1}$ dont le discours est une des dimensions structurantes.

Le travail effectué porte sur un corpus d'expositions consacrées à la génétique de 1994 à 2002. On y trouve une vingtaine d'expositions, dont certaines sont itinérantes, provenant des Centres de culture scientifiques et techniques (CCSTI), d'organismes de recherche, d'associations éducatives, du ministère de l'éducation nationale français, etc. D'une part, le travail a consisté à exploiter les archives des expositions qui n'étaient plus ouvertes au public à l'époque de nos investigations. Nous avons d'autre part analysé des expositions encore ouvertes (en France, en Belgique et en Hollande), dont nous avons photographié les expôts, archivé les textes, et interrogé les concepteurs quand c'était possible : ce sont ces dernières qui constituent le cœur de notre analyse dans le cadre de cet article. Il s'agit de :

- "L'homme et les gènes" (Cité des sciences et de l'industrie, Paris, ouverture en mai 2002).

- "Le train du génome" (multipartenariat à l'initiative de l'Institut Pasteur, et d'Aventis, France, d'octobre à novembre 2001).

- "Gènes et éthique" (Parc d'Aventures Scientifiques de Mons, Belgique).

- "Focus on genes" (Muséum de Bruxelles, de novembre 2001 à mai 2002).

- "DNA" (NEMO, Amsterdam, 2003).

- "Biodiversité" (dans le cadre de la Fête de la science, Lyon, 2002).

Dans l'état actuel des connaissances en muséologie et des documents disponibles, il est très difficile de pouvoir prétendre à

1 HeRmès n ${ }^{\circ} 25$ - Le dispositif. Entre usage et concept, Paris, CNRS éditions, 1999. 
l'exhaustivité dans la constitution d'un corpus d'expositions sur la génétique. En effet, il n'existe aucun inventaire historique des réalisations muséographiques dans le domaine des sciences et techniques. Ceci explique que nous ayons dû procéder de manière empirique, en interrogeant chaque établissement actuellement en activité ou en consultant les centres d'archives : l'histoire de la muséographie reste à faire. La plus ancienne des expositions que nous avons pu retrouver provient du Palais de la découverte, en 1946 : il s'agit de "Images de la génétique", dont le commissaire principal était Jean Rostand. On constate que le thème de la génétique, que l'on a souvent tendance à considérer comme contemporain, a tout de même fait l'objet d'une exposition internationale patronnée par l'UNESCO dans l'immédiat après-guerre. Entre 1946 et 1994, il y eut bien entendu d'autres expositions isolées ou mal documentées : nous avons donc concentré nos efforts sur la période contemporaine qui voit leur multiplication, et qui nous permet de les visiter.

Notre analyse porte sur trois dimensions énonciatives dont l'articulation va servir d'indice pour caractériser la manière dont les expositions mettent en scène le débat public et s'y inscrivent. Il s'agit tout d'abord de la représentation du débat public et de ses acteurs (médias, parole d'experts...), ensuite des différentes figures du public telles qu'elles sont mobilisées dans le discours de l'exposition, et enfin de l'implication concrète du visiteur dans le dispositif muséographique (dans le parcours général ou face à certains expôts).

\section{La représentation du débat public et de ses acteurs}

Une première observation s'impose : un certain nombre d'expositions ne font apparaitre aucun des acteurs du débat public. Elles fonctionnent dans un registre didactique en privilégiant les thématiques caractéristiques du traitement de la génétique en milieu scolaire ou universitaire : histoire des savants célèbres, mécanismes de la transmission des caractères génétiques, relations entre hérédité et environnement, formes et fonctions des chromosomes et des gènes, dysfonctionnements et maladies, techniques de la recherche médicale, agronomique ou génétique, etc.

Dans les expositions qui n'évacuent pas aussi radicalement l'existence d'un débat public, l'idée d'une transmission d'un savoir de base comme préalable nécessaire à la prise de position est récurrente. 
Il peut y avoir une diversité d'énonciateurs présents dans l'exposition, mais le débat, les polémiques, et la confrontation entre les acteurs et leurs arguments en sont absents. Par exemple, dans l'exposition "Biodiversité", les panneaux affichent les logos et discours d'un certain nombre d'institutions (Ville de Lyon, Direction Régionale de l'Environnement, Institut National de Recherche Agronomique, association de protection de la nature, etc.). Cependant, chacun de ces acteurs développe un discours consensuel et didactique, sans qu'apparaisse la moindre controverse. Le cœur des expositions sur la génétique ne porte jamais sur le débat lui-même. Quand les acteurs du débat sont présents, ils sont relativement marginalisés. Ils apparaissent en effet en périphérie de l'exposition : soit au début, soit à l'extérieur, soit à la fin, soit en toile de fond.

Au-delà de cette caractéristique commune, si l'on se concentre sur la manière dont les expositions utilisent l'espace pour construire un discours et des positions d'énonciation, on constate diverses configurations :

\section{Les médias comme introducteurs}

Dans "Le train du génome"1, le traitement du thème par la presse est présent, sur des panneaux extérieurs adossés aux wagons, lisibles par la file des visiteurs attendant de pénétrer dans l'exposition. Divers sponsors, dont Le Figaro magazine, figurent également à l'extérieur, ou sur le site web de l'exposition ${ }^{2}$. Des couvertures de magazines et des extraits sont collés sur le panneau intitulé "Le génome à la une". Lorsque le visiteur entre dans le train, il est accueilli par un moniteur TV qui diffuse en boucle un petit entretien avec Axel Kahn, en gros plan à l'écran : la parole d'un expert très présent dans les médias introduit donc à la visite sur le thème de "pour en discuter, il faut comprendre".

Dans "Focus on genes" (Muséum de Bruxelles), les médias sont présents dans l'espace de l'exposition, mais sur une mezzanine en surplomb par laquelle on descend ensuite dans l'exposition proprement dite. D'authentiques journaux, très nombreux, tapissent le mur

1 Le train du génome a circulé dans toute la France. Outre l'exposition proprement dite, visitable dans les différentes gares traversées, la production éditoriale et les animations scolaires ont été importantes.

2 http://www.traindugenome.com/ 
d'entrée, certains passages d'articles ayant été préalablement surlignées. Des magazines, ouvrages et prospectus sont disponibles sur des tables devant lesquelles on peut s'asseoir. Les visiteurs peuvent donc lire des journaux dans un espace qui n'est pas celui de la visite, mais celui d'une pratique documentaire de consultation studieuse.

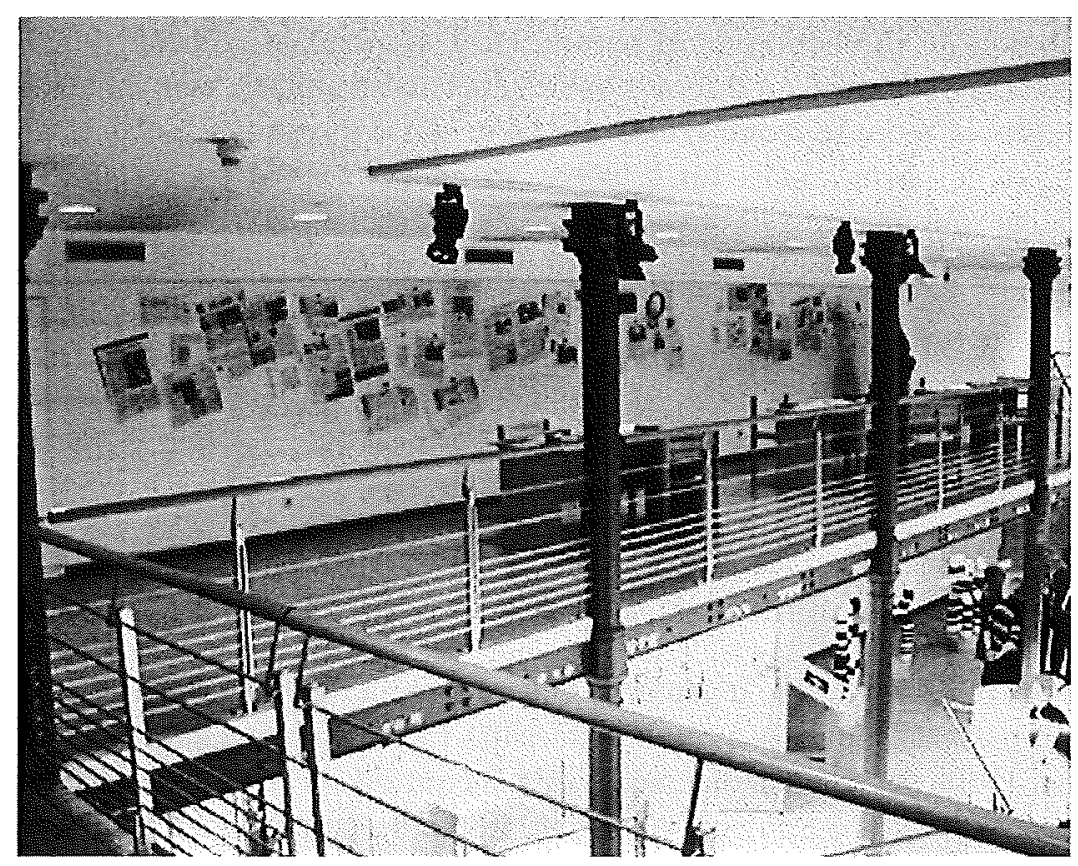

Figure 1 : La mezzanine de l'exposition "Focus on genes" 


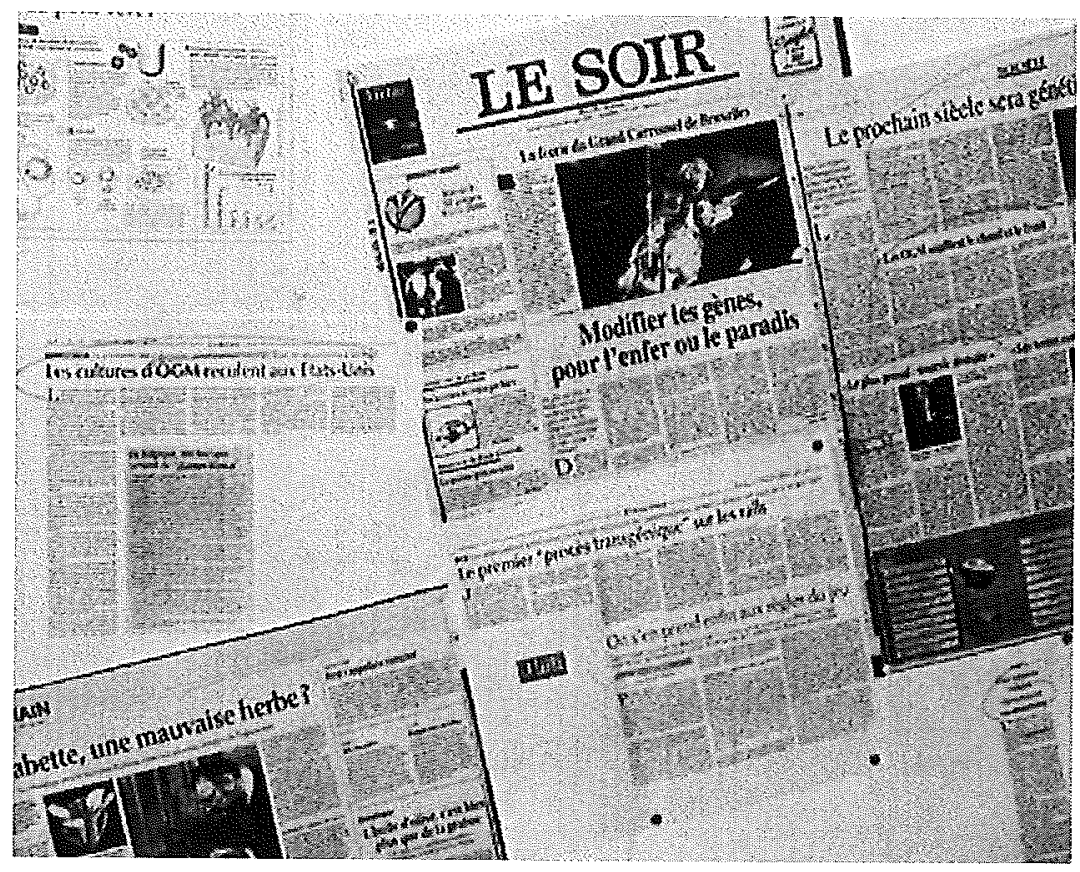

Figure 2: La revue de presse sur les murs de la mezzanine

\section{Les médias comme décors}

Dans "DNA", la presse est cette fois-ci à l'intérieur de l'exposition, représentée sous la forme d'un vrac d'images sur des panneaux de décor ceinturant des colonnes portantes. Le contenu, volontairement tronqué, ne permet pas une lecture continue : il ne s'agit pas de proposer à lire mais de simuler le foisonnement des titres de presse sur la question en exploitant un effet esthétique, celui de l'abondance et de la simultanéité de l'actualité. On retrouve là un effet exploité fréquemment à la télévision, dans les émissions à propos de science, quand il s'agit d'évoquer la couverture médiatique d'une découverte : images de journaux tournoyants, rotatives, montages de "gros titres" en une, etc. 


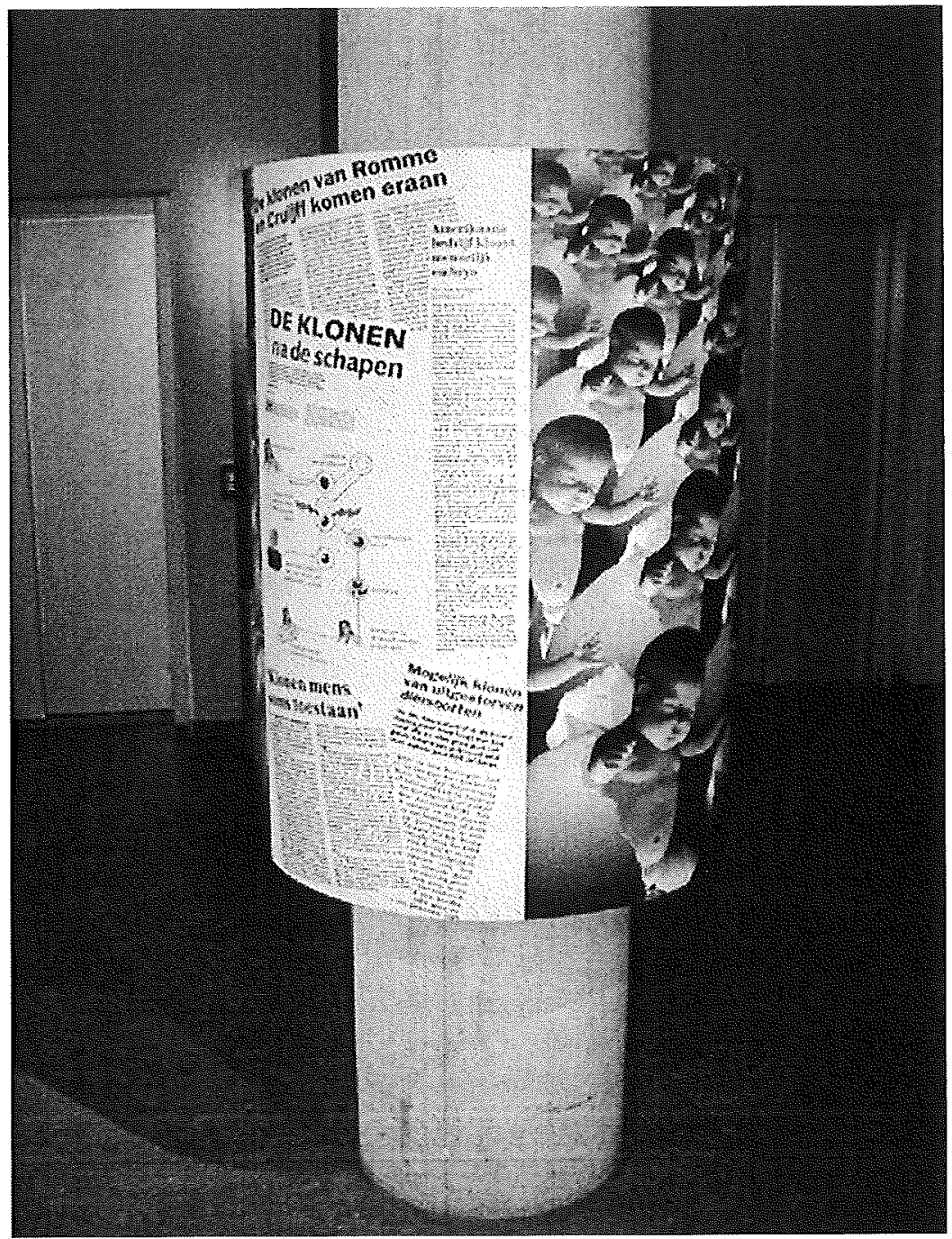

Figure 3 : un "vrac d'images" médiatiques autour d'une colonne, au NEMO

Ces figures du discours à propos de science, qu'on retrouve associées à d'autres thèmes et dans d'autres contextes (actualité, cinéma, etc.), constituent des citations qui témoignent de la conscience explicite de l'existence d'un champ médiatique, d'une 
sorte de culture commune des médias. A la télévision ${ }^{1}$ comme dans les expositions, ce type d'autoréférence ou de citation, apparaît dans la période contemporaine (les années 1990).

\section{La parole des experts comme encadrement de la visite}

Dans "Les gènes et les hommes", la presse disparaît, mais la dernière partie de l'exposition, juste avant la sortie, est entièrement consacrée à neuf experts exposant leurs points de vue. De grands panneaux comportent, sous le nom d'une personnalité, une question que celle-ci développe dans un texte, flanqué d'une courte biographie. A la fin du "Train du génome", on retrouve une interview filmée d'Axel Kahn qui invite les visiteurs à regarder le Téléthon sur France 2 et à se rendre à la Cité des sciences : l'expert désigne les liens aux autres médias, et prescrit les "bonnes" pratiques du public comme usager de l'ensemble du champ médiatique. Les visiteurs débouchent ensuite sur une série de stands animés soit par des enseignants membres de l'association des professeurs de biologie, soit par des experts d'Aventis qui est la société pharmaceutique productrice de l'exposition.

\section{La télévision comme cadre de référence du débat public}

Dans “Gènes et éthique", la dernière partie de l'exposition met en scène sous forme d'un film de fiction, une situation de choix parental et médical autour du diagnostic pré-implantatoire. Les protagonistes ont affaire à diverses instances (médecins, puis comité de bioéthique) et finissent par se tournent vers la télévision ${ }^{2}$ :

La troisième partie du débat c'est un jeu, type les jeux JeanClaude Delarue, qui ont lieu à la télévision, les débats télévisuels, à ce moment-là [les personnages] ont eu un refus, je pense, de la part des médecins, les médecins en tout cas leur demandent d'aller au niveau des conseils bioéthiques, du

\footnotetext{
I Voir I. BABOU, "Science et télévision : la vulgarisation comme construction historique et sociale", Actes du XIIe Congrès national des Sciences de l'Information et de la Communication "Émergences et continuité dans les recherches en information et en communication - UNESCO", Paris, SFSIC, 11 janvier 2001, pp. 83-91.

2 Entretien avec Christine Bluard, conceptrice de l'exposition, 3 mai 2002 à Mons.
} 
comité de consultation bioéthique, et ils font appel aux media, ils viennent exposer leur question aux media, en disant pourquoi est-ce que nous on n'aurait pas le droit ? Et à ce moment-là il y a un débat télévisé qui est organisé, et l'animateur reçoit plusieurs invités, dont une maman qui est maman d'un petit trisomique, et qui explique, si dans mon cas on m'accuse d'ici vingt ans d'avoir osé avoir un enfant trisomique, alors qu'aujourd'hui on nous donne les moyens de ne plus en avoir, qu'est-ce que je vais avoir comme recours?

Même si les références à la télévision ne sont pas très fréquentes dans notre corpus, celle-ci nous paraît très significative d'une manière de poser ce média comme lieu du débat public pour le citoyen ordinaire.

La répartition spatiale des références aux médias, généralement situés en périphérie par rapport aux expositions, témoigne d'une répartition des rôles, des statuts, voire des positions de légitimité dans le champ médiatique : le cœur des expositions s'organise autour de savoirs organisés et inscrits dans des expôts, tandis que la périphérie présente la parole foisonnante et contradictoire des médias, principalement celle de la presse.

A travers les différentes configurations repérées, les expositions n'apparaissent pas comme des espaces de mise en œuvre du débat public, même quand celui-ci est représenté. L'institution productrice de l'exposition affiche rarement une position explicitement assumée dans le débat. Or, les études de public effectuées préalablement à la programmation des thèmes de la cité des Sciences, entre 1989 à 1995 , montraient que les visiteurs s'attendaient à ce que des expositions sur le thème de l'environnement, la santé, la ville, etc., soient soustendues par une volonté de prendre pied dans le débat public sur ces thématiques. Les visiteurs interrogés anticipaient rarement la possibilité de s'exprimer directement dans l'espace de l'exposition, mais ils s'attendaient à ce que l'institution ait une volonté active d'afficher une position, en particulier par rapport aux médias, suspectés de manipuler l'opinion ${ }^{\mathrm{I}}$.

L'exposition n'est pas non plus le lieu d'accueil d'un débat public incarné dans des acteurs prenant position. Le débat est posé comme un phénomène externe, souvent esthétisé, mais qui ne débouche pas dans l'exposition sur une confrontation de points de vue

1 Voir J. Le MAREC, Le visiteur en représentations : l'enjeu des études préalables en muséologie, Thèse de doctorat, Université de Saint-Étienne, 1996. 
au sein d'une argumentation, même quand celle-ci est mimée par la coprésence des unes de presse sur les panneaux, des écrits et interviews d'acteurs du débat, et par une seule citation d'opposants rencontrée dans le corpus ${ }^{1}$. On reste dans un paradigme proche de la vulgarisation, le débat étant lui-même un "contenu" à vulgariser au plan discursif, alors même que l'exposition ne se réfère pas à l'existence d'un savoir constitué sur ce débat par les sciences humaines et sociales. Les questions et les termes du débat sont en effet cadrés, sélectionnés sans qu'une analyse du corpus de presse ne vienne justifier les choix effectués.

Enfin, l'exposition ne se constitue pas en lieu d'une diffusion de savoirs construits sur ce débat. Contrairement aux situations classiques de la vulgarisation des sciences de la nature, où l'on postule qu'un savoir complexe nécessite un travail de médiation conçu comme une opération de simplification ou de traduction, les représentations du débat public à propos de génétique font comme si ce dernier était naturellement simple et évident, à tel point qu'on peut l'exposer sans besoin de référer à des savoirs externes explicitant sa complexité.

\section{Les différentes figures du public mobilisées dans le discours}

Il est très rare dans les expositions à caractère scientifique et technique de trouver des membres du public identifiés comme tels, contrairement à la télévision qui invite régulièrement des représentants de la société civile (associations de malade, de consommateurs, d'usagers des hôpitaux, militants anti-nucléaire, etc.) ou filme des citoyens ordinaires. C'est pourtant le cas dans deux des expositions que nous avons analysées.

Dans "Le train du génome", peu avant la sortie de l'exposition, un moniteur vidéo diffuse les images d'une série d'individus (des acteurs professionnels) dont le casting est sensé représenter la diversité de la population française : le jeune, le grand-père, le maghrébin, la femme, etc. Chacun d'eux, filmé en plan poitrine sur fond neutre,

1 Dans l'exposition elle-même, un panneau est consacré aux dates marquantes du débat sur la génétique, Greenpeace apparaît pour avoir dénoncé et rendu publique l'attribution d'un brevet sur le vivant. Une photographie montre une manifestation et un slogan inscrit sur une banderole : "GATT : NO PATENTS ON LIFE". Il s'agit de la seule référence visuelle, au sein d'une exposition, au caractère parfois polémique des interventions dans le débat. 
pose une question du type : "Est-il légitime de créer des êtres humains identiques ?", "Aura-t-on dans le futur des bébés clonés ?". Ces questions sont manifestement supposées incarner les interrogations du public.

Il n'est cependant fait aucune mention des conditions dans lesquelles celles-ci auraient pu avoir été recueillies, analysées, sélectionnées. On a là affaire à un sens commun sociologique illustré par un simili-échantillon d'individus le plus varié possible au plan visuel pour représenter le "grand public" dont sont exclus tous les acteurs, y compris collectifs, qui s'expriment de fait au nom d'un métier, d'un engagement, d'une responsabilité. Ce sens commun sociologique est également à l'œuvre dans l'échantillon de questions prétendant rendre compte de la diversité et la généralité du débat public. La position simulée du public est celle du questionnement adressé aux experts par des profanes animés par un pur besoin d'informations et non par l'affirmation de valeurs ou par des conceptions de la science.

Les deux registres (l'échantillon "sociologique" et le spectre des questions) ne sont référés à aucune réalité empirique attestée. $\mathrm{Si}$ enquête il y a eu, elle n'a pas été mise en œuvre dans le sens d'un enrichissement du savoir présenté dans l'exposition'.

Ce simulacre de sociologie et d'intérêt pour le débat public est paradoxal à deux niveaux. D'une part il opère au sein de l'univers des représentations de la science comme espace de rigueur et de référence nécessaire à la vérité. D'autre part, il s'adresse à des visiteurs authentiques en chair et en os, membres effectifs d'un public qui s'incarne localement dans l'exposition. Il est surprenant que l'on ne tienne pas compte ici du contrat de communication implicite à l'exposition comme genre culturel : celui-ci fonctionne sur un double régime de valeurs : la vérité des savoirs mobilisés (le musée est une institution du savoir) et l'authenticité des objets (leur statut est précisé sur les cartels pour désigner le lien à l'univers de référence dont ils proviennent).

Dans "Gènes et éthique", on trouve un autre dispositif fictionnel intitulé "Le théâtre des controverses" qui met en scène des acteurs filmés et interprétant des situations illustrant une typologie des posi-

1 Les producteurs (Aventis et l'Institut Pasteur) ont fait publier par le journal $L e$ Monde après la fermeture de l'exposition, une pleine page de publicité mettant en avant les chiffres de fréquentation et quelques résultats d'un sondage SOFRES réalisés à la sortie du train. 
tions éthiques décrites dans un ouvrage de Dominique Mehl1 : "Le sacre de la vie", "Le sacre de la nature", "Le libéralisme scientifique", "Le libéralisme culturel", "L'éthique des droits de l'Homme" et "L'éthique de la discussion". Les situations jouées sont décrites comme "imaginaires mais qui auraient pu (et qui ont pu) parfaitement arriver"2 Par exemple, craignant d'avoir un enfant atteint de trisomie, un couple non stérile décide de concevoir un enfant par fécondation in vitro afin de permettre un diagnostic pré-implantatoire qui les prémunira de tout risque. Cette démarche n'est pas autorisée par la loi et les membres du corps médical hésitent et s'affrontent. Le théâtre des controverses, où la pièce est projetée, est une grande pièce entourée de huit écrans vidéo $(3 \mathrm{~m} \times 2,25 \mathrm{~m})$.

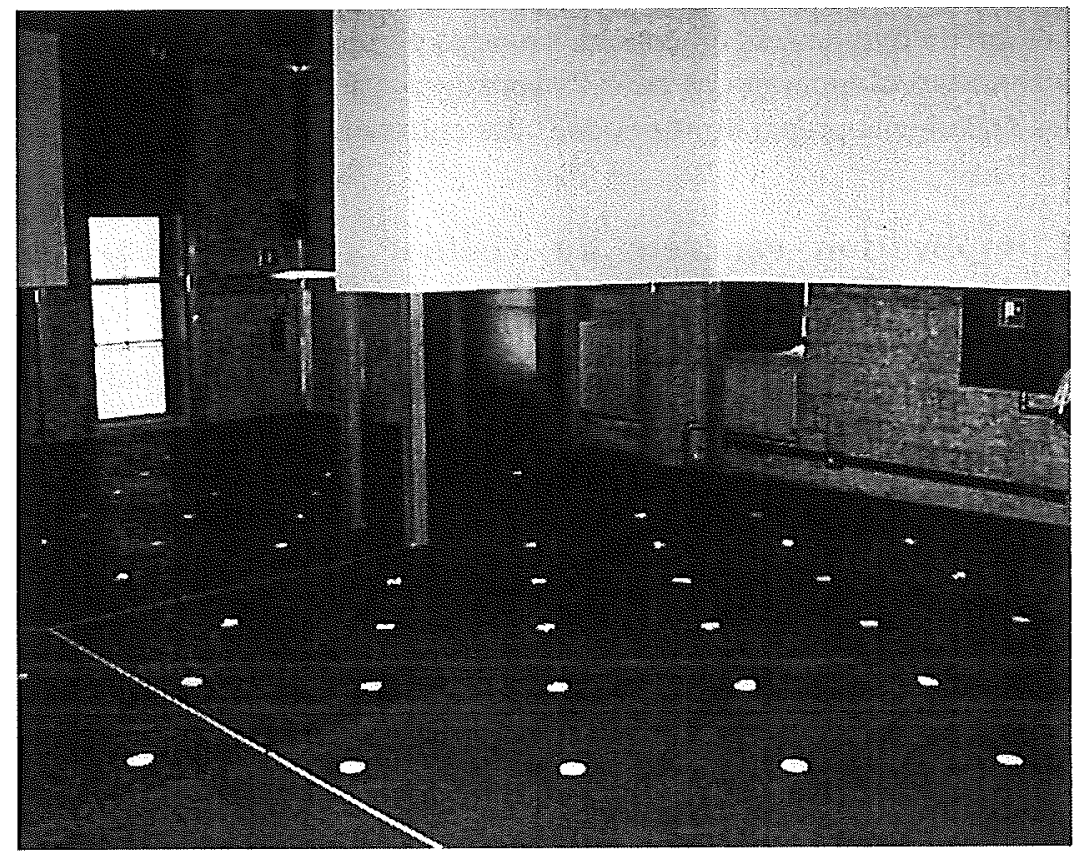

Figure 4 : Le "Théâtre des controverses".

Au sol, des capteurs enregistrent la position des spectateurs.

1 Un document d'étape de la conception de l'exposition fait explicitement référence à D. MEHL, Naître ? La controverse bioéthique, Paris, Bayard, 1999.

2 B. Babiche et V. Raisson,. Document d'étape, non publié, Mons, PASS, 17.11.2000. 
Les acteurs à l'écran ont la taille des spectateurs, et se déplacent tout autour de la pièce. Les visiteurs sont donc enveloppés par le dispositif scénique. A intervalles réguliers, ils sont sollicités pour faire évoluer le récit en condamnant ou en approuvant les arguments tenus, en se déplaçant physiquement sur l'une des trois zones actives au sol, chacune identifiée par une signalétique spécifique.

Par rapport au train du génome, la situation est un peu différente. Il y a une source sociologique qui inspire la mise en scène de questionnements et de situations : l'ouvrage de Dominique Mehl. Cependant il ne s'agit que d'une lecture effectuée par le consultant audiovisuel, et non d'une démarche systématique qui aurait été adressée aux sciences humaines par l'équipe de conception. Dominique Mehl ne figure pas dans le comité scientifique qui ne comprend que des chercheurs en sciences de la nature, et la référence à son livre n' apparaît pas dans l'exposition. Elle n'est citée que dans les documents préparatoires et dans des entretiens informels.

\section{L'implication concrète du visiteur dans le dispositif muséographique}

Dans la quasi-totalité des expositions visitées, le débat public est valorisé : il est présenté comme très ouvert et impliquant chaque citoyen. Il y a une sorte d'injonction positive à participer, qui s'adresse très directement aux visiteurs. Comment cela se traduit-il dans ce qui est proposé aux visiteurs physiquement présents? Comment organise-t-on sa participation en dehors des mises en scènes repérées plus haut?

\section{Voter avec ses pieds}

Dans le "Théâtre des controverses", le public est invité à faire progresser le scénario en choisissant à certaines étapes de celui-ci d'approuver ou de rejeter des arguments tenus par les personnages. Pour ce faire, il se déplace sur des zones actives signalées au sol. Lorsque la majorité de spectateurs se déplace sur la zone du "oui" c'est celle-ci qui détermine la suite du film. Ce dispositif est présenté comme le moment fort de l'exposition. Dans un document de programme, il est décrit comme une "expérimentation du débat". C'est "un temps pour débattre et prendre parti dans le théâtre de la 
bioéthique; il s'agit d'inviter à la réflexion sur les usages du génie génétique", les choix des spectateurs "demandent un engagement personnel, une prise de position publique"1. A la sortie, le public est informé des résultats enregistrés par les groupes précédents. Le même document explique que "cette prise de température des opinions généralement défendues est importante car elle risque d'évoluer en fonction de l'actualité". Tout se passe donc comme si le statut des opinions produites n'était lié ni à l'existence ou non d'enjeux réels (pour les votants, comme pour les acteurs à l'écran), ni à la présence d'un dispositif qui les organise, les recueille et les désigne en tant qu'opinion. L'institution muséale prend ici l'initiative de convertir les résultats du "vote" des visiteurs en un savoir sociologique, puisqu'elle le désigne, l'inscrit dans un espace dédié au savoir, le rend disponible sur une longue durée, et invite à la comparaison. Sans douter de la bonne foi des concepteurs, la naïveté du procédé trahit l'absence du caractère nécessaire d'une réflexion et d'une analyse de type sociologique sur la nature de l'opinion publique. L'analogie sociologique de la "scène" et des "acteurs" est en effet naturalisée.

\section{Le secret de l'isoloir}

Dans "Des gènes et des hommes", à la Cité des sciences, l'exposition se termine par les commentaires d'une série d'experts désignés à la fois comme auteurs et comme incarnations d'une série de positions possibles prises au nom de compétences précises. En contrepoint radical, les visiteurs sont invités à répondre anonymement, dans un isoloir doté d'une borne informatique, à une enquête par questionnaire élaborée par le CEVIPOF (Centre d'étude de la vie politique française, laboratoire associé au CNRS). Le site web de la Cité des sciences explique : "Cette dernière partie de l'exposition vous permet de mettre en perspective votre visite en vue d'un débat citoyen. Seul dans un isoloir, vous pouvez vous prononcer sur les tests génétiques". Comme dans tout sondage, le visiteur ne peut que cocher les arguments présentés dans une liste, en réponse à une question qui n'est pas forcément celle qu'il se pose à la fin de l'exposition. On peut en revanche supposer que cette enquête, validée par une instance scientifique, repose sur une typologie élaborée à partir d'une étude des

1 J.-M. Providence et C. BluARD, Gènes et éthique : programme - 17/12/02 (document de travail non publié). 
questionnements du public. Une fois le sondage achevé, au moment de donner une série d'informations sociographiques (âge, sexe, profession, etc.), le visiteur est informé que ses réponses vont servir dans le cadre d'une étude du CEVIPOF. A l'inverse du "Théâtre des controverses", le visiteur donne des informations qui seront réellement exploitées, mais dans le cadre d'un dispositif qui n'a pas été problématisé dans le discours expographique : les modalités du "débat" ainsi que les questions proposées, ont une pertinence qui relève d'un autre espace que celui de l'exposition. Comme dans le "Théâtre des controverses", il ne s'agit pas d'échanger des arguments, au risque (pour les membres du public et les experts confrontés) de devoir se déplacer ou de faire évoluer leurs positions, au risque même de constater un désaccord total. Les sciences sociales et humaines sont ici présentes explicitement, au niveau même de la conception d'un expôt : elles interviennent donc, mais à titre mineur, pour instrumentaliser à leur tour les visiteurs à l'occasion d'un sondage sur borne interactive.

\section{En file indienne : la marche incontestable du progrès}

Le train du génome a circulé dans vingt villes, au cours d'un tour de France qui a duré deux mois. Ce périple a été accompagné d'une importante couverture médiatique qui a culminé avec la parution de deux "unes" du Figaro magazine et qui précédait un Téléthon. Comme dans les grandes expositions itinérantes et pédagogiques d'avant-guerre aux États-Unis, il s'agit d'aller chercher la population partout où elle se trouve plutôt que de l'attendre dans l'enceinte d'un musée. L'intention explicite de convaincre se traduit dans la mise en scène de la générosité associée à cet effort de rapprochement. L'effet de foule, authentique ${ }^{1}$, constitue en lui-même une mise en représentation du public comme un collectif naturalisé par la coprésence, comme l'ensemble des citoyens: "Les Français veulent participer au débat scientifique et éthique"2. Le choix d'un train comme mode d'exposition y contribue largement. Cependant, dès l'entrée du train, le visiteur est sans cesse pressé d'avancer, canalisé dans le corridor étroit des wagons, par des guides interdisant tout retour en arrière. Les

1 Lors de nos deux visites, les queues à l'entrée du train étaient considérables.

2 Publi-rédactionnel pour le train du génome paru en plein page du Monde le 29.11.01. 
lampes halogènes éclairant l'ensemble du train sont d'ailleurs orientées uniquement dans le sens de la visite, rendant très difficile de se retourner pour voir l'exposition sous un autre angle que celui, frontal, de la marche du progrès. A la fin de chaque élément d'exposition, l'audio-guide désigne le suivant sur le parcours : le visiteur semble poussé en avant, tout arrêt ou recul lui étant difficilement possible. Face à ce qui ressemble à une pure opération publicitaire, il n'est guère étonnant de ne trouver aucune trace d'une référence aux sciences humaines et sociales. Mais "le train du génome" est-il même une exposition? Le fait que le visiteur ne soit pas libre de ses mouvements dans l'espace rompt avec le caractère nécessairement spatialisé de ce média, et contraint physiquement les conditions de réception.

\section{Débattre?}

Dans toutes les expositions récentes de notre corpus, il est question de l'existence d'un débat à propos des enjeux de la génétique, et les visiteurs sont invités à y participer. Il y a une sorte d'équivalence entre la représentation du public du débat tel qu'il peut se dérouler "dans la société", et le statut du public des visiteurs de ces expositions : dans les deux cas, ce public est constitué de la somme inorganisée d'individus anonymes demandeurs d'informations. Aucun des traits qualifiant habituellement l'espace public n'est présent : ni argumentation, ni échange de points de vue ne viennent troubler l'organisation d'un espace dédié à la réception, ni s'inscrire dans le discours même de l'exposition. Ce qui échappe au visiteur c'est à la fois la possibilité de questionner le cadre dans lequel on lui demande de s'inscrire pour représenter le public invité à débattre en posant des questions, mais aussi la possibilité de percevoir les acteurs, les arguments et les positionnements pourtant présents sur d'autres scènes publiques. Dans tous les cas, l'institution fait comme si elle ne participait pas au débat mais se contentait d'en créer les conditions au bénéfice du public (savoir pour comprendre et pour débattre). Ce faisant, elle masque le fait que c'est elle qui a le pouvoir (au sens d'une position de légitimité) d'orienter ce débat. Cette situation inverse les rôles et ce faisant neutralise l'expression de valeurs éloignées de la doxa. Or les études préalables menées à la Cité des sciences depuis 1990 montrent que les visiteurs enquêtés anticipent le 
fait que si une institution de ce type annonce qu'elle va traiter un thème portant sur les relations entre sciences et société (environnement, santé, etc.), c'est pour prendre position, notamment par rapport aux discours des médias, en prenant à témoin son public. Faute d'études plus systématiques sur le public, il reste bien sûr difficile de généraliser ce constat à d'autres musées ou centres de culture scientifique et technique. Cela n'empêche cependant pas d'attirer l'attention sur le crédit que le public est prêt à accorder à ce type de média, quitte à ne pas revendiquer une participation à la mode interactive ou citoyenne. Il est d'autant plus dommage d'aller au-devant d'attentes aussi fortes avec une telle incompréhension de leur sens profond, de ce type de contrat de communication qui existe entre les expositions à thème scientifiques et leur public. La convocation récurrente de la figure du "débat public", dé-problématisée par la confusion entre sondage, micro-trottoir et expression de la complexité des opinions, ressemble à un fétichisme destiné à neutraliser ce dont on parle, ce qui pose problème, ce qui oppose et ne réuni pas forcément au sein d'un consensus illusoire : quel étrange débat que celui qui ne présente aucun désaccord...

La figure du débat dans l'exposition à caractère scientifique et technique ne semble être qu'un des moyens pédagogiques pour transmettre des savoirs utiles au citoyen : comprendre comme les scientifiques serait nécessaire et suffisant pour avoir une opinion citoyenne sur les enjeux de la génétique. Il faudrait absolument commencer par comprendre la transmission des caractères génétiques, mais il n'est nul besoin d'exposer la différence entre travail sociologique et simulacre théâtral ni de comprendre le fonctionnement des médias. Pour tout ce qui relève des sciences humaines et sociales, le sens commun semble largement suffisant. Mais se contenter de déplorer l'absence inouie des savoirs issus de la recherche en sciences sociales dans ces expositions est insuffisant. Ils peuvent être sollicités très occasionnellement, mais leur intervention, ponctuelle comme dans le cas du CEVIPOF, ne remet nullement en cause des stéréotypes qui construisent le cadre global dans lequel ils s'inscrivent. Même si les sciences humaines et sociales sont fort peu sollicitées dans ce champ, il est véritablement mystérieux qu'elles ne revendiquent pas de pouvoir s'y impliquer. Tout se passe comme si les ténors des sciences sociales ne pouvaient éclairer le débat démocratique qu'en se bousculant pour commenter à n'en plus finir la télé-réalité comme modèle d'espace public idéal... 